\title{
Commentary on Campbell and Strickland (2019): Caution is needed when using self-reported alcohol use disorder screening tools
}

\author{
Stéphanie Baggio ${ }^{\mathrm{a}, \mathrm{b}, *}$, Katia Iglesias ${ }^{\mathrm{c}}$ \\ ${ }^{a}$ Division of Prison Health, Geneva University Hospitals and University of Geneva, Chemin du Petit Bel Air 2, 1226 Thônex, Switzerland \\ ${ }^{\mathrm{b}}$ Department of Forensic Psychiatry, Institute of Forensic Medicine, University of Bern, Falkenplatz 16, 3012 Bern, Switzerland \\ ${ }^{\mathrm{c}}$ School of Health Sciences, HES-SO University of Applied Sciences and Arts of Western Switzerland, Route des Arsenaux $16 a, 1700$ Fribourg, Switzerland
}

\section{H I G H L I G H T S}

- There is a lack of well-validated screening tools for alcohol use disorder.

- Screening tools should be validated against a gold standard to provide evidence of their validity.

- Caution is needed when using screening tools as the false-positive rate is very high.

The study of Campbell and Strickland (2019) addresses a very important research question: the lack of reliable and valid short self-reported screening tools designed to identify alcohol use disorder (AUD) in community samples. The authors concluded that the Brief AUD-5 diagnostic assessment is an "efficient, reliable, and valid self-report tool" (p. 196). Given the methodology of the study, we believe that this conclusion is overstated. The authors assessed between-items reliability and convergent validity with self-reported alcohol use measures and a well-known self-reported screener, namely the Alcohol Use Disorder Identification Test (AUDIT, de Meneses-Gaya, Zuardi, Loureiro, \& Crippa, 2009; Lundin, Hallgren, Balliu, \& Forsell, 2015). Unfortunately, this method does not address previous flaws met by self-reported AUD screeners. Indeed, a frequent issue of such screeners is their lack of specificity and consequently, their important number of false positives (Maraz, Király, \& Demetrovics, 2015). It means that some -or even many-individuals who screen positive do not have the disorder. With a prevalence rate of $36.1 \%$ of AUD, probably due to the sample selection (a subset of individuals who were positive on the brief screener), this pitfall might have occurred in the sample. In a recent study, we showed that a self-reported AUD screener assessing the eleven symptoms of the DSM-5 had a 54.2\%-rate of false positives using the recommended threshold of two symptoms or more (Baggio et al., 2018; Iglesias, Sporkert, Daeppen, Gmel, \& Baggio, 2018). In our study, the AUDIT also displayed a high rate of false positives (47.1\%). Sensitivity should be prioritized in clinical settings, when the aim is to detect individuals with potential AUD and when used before a complete clinical assessment. However, in research and public health planning, this second step never -or rarely- occurs, and we believe that a high rate of false positives is simply misleading. Therefore, we think that concluding on the reliability and validity of the Brief DSM-5-AUD for "diverse research and clinical settings" (p. 194) is overstated.

A second notable issue was that alcohol use was probably not the better choice to assess convergent validity. Indeed, we should keep in mind that alcohol use only presents a moderate overlap with AUD (Rehm et al., 2013; Saha, Stinson, \& Grant, 2007) and that AUD does probably not only reflect a magnitude of use. Even if some thresholds for "safer" alcohol use are available, they have been tested against health problems (Department of Health, 2016; Rehm, 2016; Wood et al., 2018).

Today, it is well-established that symptoms of AUD constitute a unidimensional construct with meaningful severity-related categories, moderately correlated with alcohol use. What we really need is the proof of screeners' validity against clinical diagnoses and empirical evidence to support the thresholds defining AUD among different subgroups of the population (e.g., young people, non-clinical populations, males and females). The only way to decide whether a screening tool is valid in this way is to compare it to a gold standard. Six years after the release of the Diagnostic and Statistical Manual for Mental Disorders DSM-5 (APA, 2013), there is still a dearth of studies on this topic and empirical evidence is crucially needed. In the case of alcohol, the gold standard should be a clinical diagnosis (ideally a semi-structured interview conducted by an experienced clinician). Without such methodology, it is very difficult to conclude that a screening tool is valid and thus, using cut-off scores should be avoided.

Therefore, even if the Brief AUD-5 assessment displayed acceptable internal consistency and relations with variables of the field, researchers and clinicians should be aware that this is not a screening tool designed to classify drinkers as AUD- or non-AUD. In the absence of

\footnotetext{
* Corresponding author at: Division of Prison Health, Geneva University Hospitals and University of Geneva, Chemin du Petit Bel Air 2, 1226 Thônex, Switzerland.

E-mail addresses: stephanie.baggio@hcuge.ch (S. Baggio), katia.iglesias@hefr.ch (K. Iglesias).
} 
convincing evidence on the psychometric performance of the Brief AUD-5 assessment with regards to a gold standard, it would even be more accurate to consider it as a continuous or count variable indicating a severity of AUD rather than a diagnosis. This is in line with the recent switch which assumes that disorders are dimensional constructs rather than categorical outcomes (Kerridge, Saha, Gmel, \& Rehm, 2013; Liu, 2017). Unfortunately, many studies use a similar design and conclude about the relevance of AUD screeners based on insufficient methodologies. Such conclusions are very misleading for future research and clinical practice and thus it is important to raise awareness on this severe flaw in current alcohol research.

\section{Funding}

This commentary did not receive any specific grant from funding agencies in the public, commercial, or not-for-profit sectors.

\section{Declaration of Competing Interest}

The authors declare no competing interest.

\section{References}

APA (2013). Diagnostic and statistical manual of mental disorders (5th ed.). Arlington, VA: American Psychiatric Publishing.

Baggio, S., Rothen, S., Sporkert, F., Daeppen, J.-B., Gmel, G., \& Iglesias, K. (2018). Selfreported DSM-5 eleven criteria to assess alcohol use disorder: Is it a reliable and accurate brief assessment? Evidence from a community-based sample, proceedings of the 19th world congress of International Society for Biomedical Research on Alcoholism (ISBRA2018), Kyoto, Japan, September 9-13 2018. Alcoholism, Clinical and Experimental Research, 42(S2), 133A.

Campbell, E. M., \& Strickland, J. C. (2019). Reliability and validity of the brief DSM-5 alcohol use disorder diagnostic assessment: A systematic replication in a crowdsourced sample. Addictive Behaviors, 92, 194-198.

Department of Health (2016). Alcohol guidelines review - report from the guidelines development group to the UK chief medical officers. London, UK: Department of Health.

Iglesias, K., Sporkert, F., Daeppen, J.-B., Gmel, G., \& Baggio, S. (2018). Comparison of self-reported measures of alcohol-related dependence among young Swiss men: A study protocol for a cross-sectional controlled sample. BMJ Open, 8(7), e023632.

Kerridge, B. T., Saha, T. D., Gmel, G., \& Rehm, J. (2013). Taxometric analysis of DSM-IV and DSM-5 alcohol use disorders. Drug and Alcohol Dependence, 129(1-2), 60-69.

Liu, R. T. (2017). Substance use disorders in adolescence exist along continua: Taxometric evidence in an epidemiological sample. Journal of Abnormal Child Psychology, 45(8), 1577-1586.

Lundin, A., Hallgren, M., Balliu, N., \& Forsell, Y. (2015). The use of alcohol use disorders identification test (AUDIT) in detecting alcohol use disorder and risk drinking in the general population: Validation of AUDIT using schedules for clinical assessment in neuropsychiatry. Alcoholism: Clinical and Experimental Research, 39(1), 158-165.

Maraz, A., Király, O., \& Demetrovics, Z. (2015). Commentary on: Are we overpathologizing everyday life? A tenable blueprint for behavioral addiction research. The diagnostic pitfalls of surveys: If you score positive on a test of addiction, you still have a good chance not to be addicted. Journal of Behavioral Addictions, 4(3), 151-154.

de Meneses-Gaya, C., Zuardi, A. W., Loureiro, S. R., \& Crippa, J. A. S. (2009). Alcohol use disorders identification test (AUDIT): An updated systematic review of psychometric properties. Psychology \& Neuroscience, 2(1), 83-97.

Rehm, J. (2016). How should prevalence of alcohol use disorders be assessed globally? International Journal of Methods in Psychiatric Research, 25(2), 79-85.

Rehm, J., Marmet, S., Anderson, P., Gual, A., Kraus, L., Nutt, D. J., ... Gmel, G. (2013). Defining substance use disorders: Do we really need more than heavy use? Alcohol and Alcoholism, 48(6), 633-640.

Saha, T. D., Stinson, F. S., \& Grant, B. F. (2007). The role of alcohol consumption in future classifications of alcohol use disorders. Drug and Alcohol Dependence, 89(1), 82-92.

Wood, A. M., Kaptoge, S., Butterworth, A. S., Willeit, P., Warnakula, S., Bolton, T., Danesh, J. (2018). Risk thresholds for alcohol consumption: combined analysis of individual-participant data for 599912 current drinkers in 83 prospective studies. The Lancet, 391(10129), 1513-1523. 